Bio-grafía Escritos sobre la Biología y su Enseñanza.

Edición Extra-Ordinaria.

Memorias del I Congreso Nacional de Investigación en Enseñanza de la Biología.

VI Encuentro Nacional de Investigación en Enseñanza de la Biología y la

Educación Ambiental. ISSN 2027 1034. P. P. 560-586.

\title{
ENSEÑANZA DE LA BIOLOGÍA EN UN PAÍS BIODIVERSO, PLURIÉTNICO Y MULTICULTURAL. APROXIMACIONES EPISTEMOLÓGICAS.
}

POR: Norma Constanza Castaño Cuéllar ${ }^{1}$.

\section{RESUMEN}

Las condiciones contemporáneas exigen un pensamiento crítico y transformador, alrededor de aspectos económicos, políticos y culturales en torno a la globalización cultural en la que estamos inmersos y ello requiere también de elementos para la formación de ciudadanía, más cuando se trata del uso de factores naturales en ecosistemas tan importantes biológica, política y económicamente como lo son los de este país.

Se requieren otras formas de apropiación del conocimiento biológico y, por ende, formas diferentes de pensar la educación en el país, siendo vital el compromiso con las realidades concretas desde la formación de maestros, a partir de la comprensión de las particularidades del contexto y de los vínculos con las poblaciones, en la perspectiva de trascender con sentido las posibilidades de vida de las personas y de las comunidades

Los retos de la educación en biología, están en establecer relaciones entre las formas políticas contemporáneas, la educación y la búsqueda de alternativas posibles en contexto. de lo que somos - nosotros y los otros. Gestar propuestas alternativas que involucren a todos los sectores de la sociedad, como un ejercicio de libertad y solidaridad humana. Abordar la globalización, el posdesarrollo y la sustentabilidad ecológica, en formas social y políticamente efectivas.

Para lograrlo es fundamental auto reconocernos como sujetos de poderes: reconocernos en y desde la diferencia evita anular la mirada del otro y también rompe la inercia de colonizar epistemológicamente a las culturas y grupos humanos definidos en la alteridad; perder las certezas y ganar libertades, no exponernos y exponer a los distintos tipos de violencias que genera la

\footnotetext{
1 Docente investigadora Universidad Pedagógica Nacional - Departamento de Biología. Coordinadora Especialización en Enseñanza de la Biología. Estudiante Doctorado Interinstitucional en Educación Línea de investigación Educación en Ciencias en contextos culturales. Universidad Distrital Francisco José de Caldas. constanza castano@yahoo.es
} 
Bio-grafía Escritos sobre la Biología y su Enseñanza.

Edición Extra-Ordinaria.

Memorias del 1 Congreso Nacional de Investigación en Enseñanza de la Biología.

VI Encuentro Nacional de Investigación en Enseñanza de la Biología y la

Educación Ambiental. ISSN 2027 1034. P. p. 560-586.

reproducción sin sentido y la homogenización de una sola forma de concebir el mundo.

Palabras clave: Enseñanza de la biología, interculturalidad, relación vida y vivo.

\section{ABSTRACT}

The contemporary conditions require critical and transformative thinking, about economic, political and cultural globalization around the culture in which we are involved and this element also requires the formation of citizenship, especially when it comes to the use of natural factors biological ecosystems as important, politically and economically as are those in this country.

They require other forms of appropriation of biological knowledge and therefore different ways of thinking about education in the country and is vital engagement with the concrete realities from the training of teachers, from the understanding of the particularities of the context and ties with populations in transcending the prospect of meaningful life chances of individuals and communities.

The challenges of education in biology, are to establish relationships between contemporary political forms, education and the search for alternatives in context. of who we are - we and the others. Take shape alternative proposals that involve all sectors of society, as an exercise of freedom and human solidarity. Addressing globalization, post-development and ecological sustainability in social and politically effective ways.

To achieve this fundamental self-recognition as subjects of powers in and from recognizing the difference override prevents other's eyes and break the inertia of epistemological colonizing cultures and groups defined in otherness, losing the certainties and win freedom, not exposed and exposed to different types of violence that generates reproduction and homogenization meaningless one way of conceiving the world.

Keywords: teaching of biology, interculturalism, relationship life and living.

\section{INTRODUCCIÓN}

Colombia se considera hoy un país pluriétnico y multicultural; este reconocimiento se inició apenas en la década del setenta, cuando las organizaciones indígenas movilizaron un proceso de reafirmación y conciencia de su identidad que dio como 
Bio-grafía Escritos sobre la Biología y su Enseñanza.

Edición Extra-Ordinaria.

Memorias del 1 Congreso Nacional de Investigación en Enseñanza de la Biología.

VI Encuentro Nacional de Investigación en Enseñanza de la Biología y la

Educación Ambiental. ISSN 2027 1034. P. p. 560-586.

resultado que la multiculturalidad del país fuera reconocida en la Constitución Nacional de 1991.

Con la Constitución de 1991, los derechos fundamentales de los grupos étnicos se han afianzado y ha surgido una nueva relación con el Estado colombiano a partir de la aceptación de la diversidad cultural: "El gran agregado de la Constitución de 1991 fue la concreción y expresión normativa de la necesidad de fomentar en toda la sociedad relaciones de mutualidad e interculturalidad, en vez de las de dominación de la sociedad hegemónica hacia las minorías étnicas" (Bodnar, 2005:9). Este reconocimiento hace surgir un nuevo tipo de relación entre el Estado y su población frente a la diversidad étnica, pasando de relaciones de tipo vertical a relaciones horizontales con grupos de población considerados como minoritarios, lo que genera la necesidad de asumir un enfoque diferencial que valore y reconozca la diversidad. Entre este reconocimiento se manifiesta el derecho a una formación que respete y propicie su identidad cultural (Art. 68); ello implica desarrollar procesos educativos que hagan visibles a estos grupos étnicos, para que sus reivindicaciones y sus necesidades de participación puedan ser atendidas debidamente por el estado.

Desde otra perspectiva, sabemos que las tendencias globalizadoras contemporáneas no se circunscriben al aspecto económico, sino que también inciden en el desconocimiento de los saberes y la falta de reconocimiento de la diversidad territorial y cultural. Esto generalmente se traduce en políticas públicas educativas reducidas a planteamientos homogenizantes alrededor de las competencias para el trabajo y lo productivo, lo que genera exclusión de diversas formas, particularmente de los derechos sociales, negando incluso el acceso al conocimiento a las comunidades, especialmente en las regiones con alta diversidad cultural.

Las condiciones contemporáneas exigen un pensamiento crítico y transformador, alrededor de aspectos económicos, políticos y culturales en torno a la globalización cultural en la que estamos inmersos y ello requiere también de elementos para la formación de ciudadanía, más cuando se trata del uso de factores naturales en ecosistemas tan importantes biológica, política y económicamente como lo son los de este país.

Se requieren otras formas de apropiación del conocimiento biológico y, por ende, formas diferentes de pensar la educación en el país, siendo vital el compromiso con las realidades concretas desde la formación de maestros, a partir de la comprensión de las particularidades del contexto y de los vínculos con las poblaciones, en la perspectiva de trascender con sentido las posibilidades de vida de las personas y de las comunidades 
Bio-grafía Escritos sobre la Biología y su Enseñanza.

Edición Extra-Ordinaria.

Memorias del I Congreso Nacional de Investigación en Enseñanza de la Biología.

VI Encuentro Nacional de Investigación en Enseñanza de la Biología y la

Educación Ambiental. ISSN 2027 1034. P. p. 560-586.

De acuerdo con Lee y Luyks (Citado en: Abell y Lederman. 2008, p. 171 - 191) desde una óptica anglosajona, el conocimiento en ciencia y tecnología es cada vez más importante en el mundo actual: existe un creciente número de profesiones que requieren familiarizarse con los conceptos científicos y herramientas de alta tecnología para tomar decisiones basadas en el conocimiento científico. Igualmente se está incrementando la diversidad de la población en edad escolar, junto con un rendimiento diferencial de la ciencia entre los grupos poblacionales, lo cual hace que el objetivo "ciencia para todos" se constituya en un reto para muchas naciones.

Con demasiada frecuencia, afirman Lee y Luyks, las investigaciones acerca del conocimiento sobre los profesores de ciencia y la diversidad es insuficiente para guiar a los estudiantes de diversos orígenes hacia el aprendizaje significativo de las ciencias y tendencias como la estandarización de los currículos y su valoración estándar van en contra de la equidad (McNeil, 2000).

La estandarización de las medidas para alcanzar logros científicos han puesto de manifiesto importantes lagunas entre estudiantes de diversos orígenes raciales, étnicos y socioeconómicos. Aunque se ha avanzado en las últimas décadas, las ganancias han sido pocas en relación con las inequidades que aún siguen persistiendo. Las preguntas para docentes e investigadores se relacionan con ¿qué es lo que constituye unas oportunidades equitativas de aprendizaje? ¿Cómo pueden variar esas oportunidades para diferentes poblaciones de estudiantes? ¿Cómo pueden contribuir en contextos con recursos limitados y en situaciones en las que hay conflictos en cuanto a las prioridades educativas?

Enfocarse sobre la diversidad de los estudiantes que ingresan a la escuela, presume que deben seleccionarse aspectos relacionados con el currículo, las prácticas y la organización escolar, que afectan directamente a poblaciones estudiantiles diversas. Por lo tanto, los resultados en la enseñanza de las ciencias, pueden ser producto de la manera en que las políticas y las escuelas definen, delimitan y gestionan la diversidad del alumnado, como también de la diversidad en sí misma.

Independientemente del origen o la naturaleza de las marginaciones sobre los estudiantes, el éxito académico a menudo depende de la asimilación de las normas convencionales. En el caso de los inmigrantes o de estudiantes nacidos en USA, que constituyen minorías raciales/étnicas, depende en gran medida del grado de adquisición del lenguaje estándar y de su adaptación a la cultura común de la sociedad dominante en USA. Por ejemplo, tradicionalmente en la instrucción en ciencias, se asume que los estudiantes tienen ciertos recursos educativos en sus hogares y que los estudiantes pobres necesitan adoptar hábitos de aprendizaje, que requieren cierto nivel de estabilidad económica. 
Bio-grafía Escritos sobre la Biología y su Enseñanza.

Edición Extra-Ordinaria.

Memorias del 1 Congreso Nacional de Investigación en Enseñanza de la Biología.

VI Encuentro Nacional de Investigación en Enseñanza de la Biología y la

Educación Ambiental. ISSN 2027 1034. P. P. 560-586.

Afirman Lee y Luyks (Citado en: Abell y Lederman, 2008) que la interacción raza/etnicidad, cultura, lenguaje y clase social, es compleja. Por una parte es difícil metodológicamente separar las influencias de distintas variables, que atraviesan a esas poblaciones, de modo que no son fáciles de dilucidar (por ejemplo, cuando los grupos étnicos están internamente estratificados en clases). De otro lado, estas variables no son enteramente separables, conceptualmente hablando; el lenguaje es un importante elemento de etnicidad, la cultura está en parte determinada por la clase social y así sucesivamente. Las identidades raciales/étnicas y las competencias lingüísticas están menos limitadas de lo que implica normalmente el uso de categorías demográficas; ellas pueden variar en un solo hogar o aún más en un individuo, dependiendo de las situaciones. Aún más, aunque el lenguaje, la cultura y la raza son componentes importantes de la identidad colectiva étnica, la relativa importancia de cada componente varía de un grupo a otro.

En esta perspectiva es necesario recordar el sistema educativo pone en práctica una singular acción, interrelacionada con las prácticas de la clase dominante y su cultura, provee de cierta información que es capaz de ser aprehendida sólo por aquellos sujetos que poseen el sistema de predisposiciones que es condición para el éxito en la transmisión de la cultura que (Bourdieu y Passeron, 2005). Bernstein (1990) también nos recuerda que la escuela tiene claramente definidos los roles y las reglas de comportamiento, requiriendo que los participantes dispongan de códigos lingüísticos específicos para identificar y desempeñar los roles y las conductas esperadas ${ }^{2}$, es así como la escuela se convierte en un dispositivo de poder que ordena y jerarquiza.

La escuela a través del sistema de enseñanza de los conocimientos impone simbolismos y significados sobre grupos o clases de modo que tal imposición se concibe como legítima, es lo que Bourdieu denomina violencia simbólica (Citado en: Gutierrez, 2004, pag. 290).: "...un concepto articulador de diferentes fenómenos sociales que afectan especialmente a los distintos ámbitos de producción de sentido. Es decir, está presente en cada uno de los campos donde circulan y se disputan entre los agentes sociales comprometidos en esos juegos (el campo escolar evidentemente, pero también el campo político, el campo artístico, el campo intelectual, el campo de las clases sociales, etc.), los bienes simbólicos que allí están en juego, en el marco de estructuras de posiciones y de relaciones entre posiciones de dominación-dependencia"

Tal legitimidad oculta relaciones de poder, lo que permite que la imposición sea eficaz. En la medida en que es aceptada como legítima, la cultura añade su propia

\footnotetext{
${ }^{2}$ Bernstein distinguió entre contextos comunicacionales especializados y no especializados. Un contexto comunicacional especializado es un contexto fuertemente clasificado, esto es, un contexto en el cual la interacción está vinculada con la situación o los principios espaciales muy estrechamente.
} 
Bio-grafía Escritos sobre la Biología y su Enseñanza.

Edición Extra-Ordinaria.

Memorias del 1 Congreso Nacional de Investigación en Enseñanza de la Biología.

VI Encuentro Nacional de Investigación en Enseñanza de la Biología y la

Educación Ambiental. ISSN 2027 1034. P. p. 560-586.

fuerza a las relaciones de poder, contribuyendo a su reproducción sistemática, generando arbitrariedad en su imposición y en su contenido. Parafraseando a Gutierrez (2004), el poder es ontológico, existe en las cosas y en los cuerpos, en los campos y en los habitus, en las instituciones y en los cerebros; tiene múltiples dimensiones: existe físicamente, objetivamente y simbólicamente.

La violencia simbólica en la acción educativa, surge especialmente de la didáctica que se fundamenta en la imposición de la autoridad (método o modelo pedagógico didáctico) y del conocimiento que se enseña como verdadero, definitivo, acabado, y en consecuencia en forma ahistórica y acrítica.

En este sentido, se plantea que los conocimientos se producen en el campo de las llamadas "ciencias puras" y la didáctica pasa de construir un campo conceptual (en el sentido de Bourdieu) a constituir una disciplina: de definir unos problemas a generar unas prácticas en las que se atraviesan "juegos de poder" y en las que el propósito es el aprendizaje (Castaño, 2010).

La didáctica encierra la realidad del saber escolar: formas de entendimiento o de comprensión; una forma de narración del conocimiento; un dispositivo que prepara para el ejercicio futuro de la ciencia. Se ubica en un plano opuesto a la pedagogía: dado que la ciencia constituye una prioridad para la sociedad, desde la didáctica se propone incentivar y formar las próximas generaciones y así las ciencias se constituyen en un campo de poder; especialmente de aquellas ciencias que contribuyen al fortalecimiento del capitalismo en la funcionalización que este hace del conocimiento para su propio beneficio (Alban, 2010).

Las preguntas del pedagogo, se alude, son otras ¿para qué educamos? ¿Cómo hacer libres y felices a los niños? ¿Cómo subvertir el orden de la razón? "El pedagogo es un filósofo del sufrimiento, del aprender, un político de la resistencia y un subversivo de los sistemas de escolarización" afirma Zambrano (2010). La pedagogía es una forma de resistencia; pensar en el aprender es preocuparse por el sujeto y el lugar que ocupa en la sociedad; pensar en el enseñar es convertir la didáctica en una metodología y esto la ubica en el marco de la razón. ¡La didáctica es un conocimiento cartesiano! hoy en tiempos de globalización, en general, la didáctica se concibe más desde el logro de los fines de la educación en las distintas disciplinas, al punto que se privilegian los resultados desde una ideología de la eficacia (Camilloni, \& Basabe \& Feeney, 2007).

La perspectiva de la didáctica como poder, impone saberes y los normaliza para regular; pero también genera discursividad y disposición. Es decir los saberes constituyen un universo sociológico; nos apropiamos de ellos para dominar. 
Bio-grafía Escritos sobre la Biología y su Enseñanza.

Edición Extra-Ordinaria.

Memorias del 1 Congreso Nacional de Investigación en Enseñanza de la Biología.

VI Encuentro Nacional de Investigación en Enseñanza de la Biología y la

Educación Ambiental. ISSN 2027 1034. P. p. 560-586.

Todas las culturas cuentan con arbitrariedades culturales que son transmitidas a través de los procesos de socialización, disfrazados de legitimidades no cuestionadas (la imposición ha de ser vista como independiente de las relaciones de fuerza) y como cuestión meramente técnica, he aquí otra vez la didáctica.

Para Bourdieu (sf) la escuela, ejerce una violencia simbólica al imponer a los hijos de las clases dominadas ese arbitrario cultural: el currículo como universal y necesario como si fuese "la cultura", al mismo tiempo que convierten en ilegitimas otras formas de cultura propias; estableciendo una distinción entre los conocimientos legítimos y dominantes y otros conocimientos subordinados. El sistema educativo no sólo permite esa reproducción de las desigualdades a través de la legitimación eufemística, sino que incrementa las desigualdades de partida. Se subraya el hecho de que la cultura escolar no es neutral, ya que enmascara relaciones de dominación que contribuyen a reproducir las desigualdades de clase, a través del discurso sostenido por la didáctica.

Desde estas perspectivas es evidente que desde el campo de la educación se ha impuesto la primacía de los saberes occidentales, lo cual pone en condición de subalternidad a saberes surgidos en otras condiciones culturales.

Parece necesario ocuparse de otros conceptos, que involucren al sujeto y que podrían contribuir a la comprensión de nuestras propias problemáticas educativas, especialmente las que se suscitan en torno a la formación y a la diversidad cultural.

El trabajo académico implica una responsabilidad ética. Es importante tener en cuenta desde dónde se analiza, conocer el origen y el contexto de los conceptos, reconocer la fragmentación del conocimiento y ver cómo todos hacen parte de una misma cosmovisión, es decir, una cosmovisión universalista, liberal y occidental, impuesta violentamente desde los procesos coloniales modernizadores.

Si se considera al campo de la educación como una labor pedagógica y en beneficio de la sociedad en su conjunto, se tienen que elaborar propuestas, críticas y alternativas, pues ya lo plantea Leff $(1986$, p. 36) "el conocimiento no se constituye independientemente ni se da en un vacío ideológico"

En la educación raras veces se cuestiona el conocimiento en sí, se considera esto una labor más bien perteneciente a la filosofía, pero resulta ser un elemento demasiado importante como para obviarlo, al ser justamente el conocimiento el que se está transmitiendo. El profesor es el multiplicador y facilitador de tal conocimiento y al cumplir una labor de semejante responsabilidad resulta indispensable, que tenga una conciencia y un entendimiento claro sobre los orígenes, los contextos y las implicaciones del conocimiento que está trasmitiendo. 
Bio-grafía Escritos sobre la Biología y su Enseñanza.

Edición Extra-Ordinaria.

Memorias del I Congreso Nacional de Investigación en Enseñanza de la Biología.

VI Encuentro Nacional de Investigación en Enseñanza de la Biología y la

Educación Ambiental. ISSN 2027 1034. P. P. 560-586.

La gradual reformulación de lo pedagógico, confluye con la centralidad que los factores de orden cognitivo presentan en los modelos políticos y económicos globales, lo que a su vez puede explicar el rol atribuido a la educación en el desarrollo multidimensional de los países.

\section{LA ENSEÑANZA DE LA BIOLOGÍA EN COLOMBIA}

En una investigación realizada por Chona \& Castaño \& Cabrera (1998), se plantea que la enseñanza de la Biología en Colombia ha sido influenciada por elementos de poder y de saber provenientes de diferentes instituciones normalizadoras. Los enfoques con los que se ha abordado la enseñanza, provienen más de la estructuración de elementos políticos, administrativos y normativos que del establecimiento de criterios sobre lo que debe ser la función de la enseñanza de la Biología.

Los estudios sistemáticos sobre la historia de la enseñanza de la Biología en Colombia son escasos afirman "vislumbramos que la enseñanza de la Biología en Colombia se encuentra mezclada bajo diferentes enfoques en los ámbitos sociales, políticos y económicos. La concepción de enseñanza es aún confusa y en el orden educativo hemos podido detectarla dispersa o refundida en conceptos como pedagogía y didáctica..." (Chona, 1998, p. 6-7). Enfoques con los que se ha abordado la enseñanza, provienen más de la estructuración de elementos políticos, administrativos y normativos, que del establecimiento de criterios sobre lo que debe ser la función de la enseñanza de la Biología.

Es así como en un principio, los conocimientos llegados al denominado Nuevo Mundo se centran en la instrucción de la historia natural y de la filosofía. Las comunidades religiosas son entonces los centros de difusión y en consecuencia, los preceptores son españoles en su mayoría. Las ideas, pensamientos y hechos fueron cohesionados, correlacionados y refundidos en el contexto latinoamericano, pues al tener una sola óptica, la de los colonizadores, desde allí se interpretan, narran y establecen los elementos de conocimiento y crítica de las realidades (Rodríguez, citado en Chona, 1998).

En un segundo período, los conocimientos provenientes de las Ciencias Naturales, fueron incorporados a la vida universitaria en las carreras de Medicina, Jurisprudencia y Filosofía. Las Ciencias Naturales también presentan un momento específico en la historia colombiana con la creación de las Escuelas de Ciencias Naturales de la Universidad Nacional. Hacia 1868 se crean distintas cátedras en la Escuela y es importante destacar que ninguna se denominó Biología; se trataba 
Bio-grafía Escritos sobre la Biología y su Enseñanza.

Edición Extra-Ordinaria.

Memorias del 1 Congreso Nacional de Investigación en Enseñanza de la Biología.

VI Encuentro Nacional de Investigación en Enseñanza de la Biología y la

Educación Ambiental. ISSN 2027 1034. P. p. 560-586.

de cursos de Botánica, Zoología, Anatomía Comparada, entre otros (Restrepo,1984).

A finales del Siglo XIX surgen en el ámbito universitario polémicas en torna al concepto vida como lo constata la obra del Padre Luis Ortiz en 1893 y el Estudio sobre el Sistema Evolucionista de Emilio Cuervo (1891, citados en: Chona, 1998).

Paralelas al desarrollo de estas polémicas, encontramos cátedras de Biología en la Universidad Republicana, en el Externado de Colombia y en la Facultad de Filosofía de la Universidad del Rosario. Las cátedras son transitorias y en ellas se discute en torno a las teorías sobre el origen de la vida que venían formulándose y a la teoría celular, reforzada con la experimentación sobre la naturaleza orgánica de los seres vivos, como lo evidencia la obra del Padre Ortiz, ya mencionada.

El establecimiento del Concordato entre la Iglesia Católica y el Estado Colombiano, hace que se controle la difusión de obras científicas, lo cual no permite el desarrollo de las cátedras de Biología, máxime que su temática era ofrecida a juristas, médicos y letrados, como trabajo de discusión ideológico y no de análisis experimental (Restrepo, 1984).

Como es notorio, la Biología aún no encuentra un ámbito institucional firme para su consolidación como práctica científica, pero no por esto desaparece de la historia colombiana. Toma un nuevo papel en la polémica sobre la degeneración de la raza hacia 1920. La Biología participa con su saber con el objeto de evaluar la condición de la población y su posible estado de degeneración y decadencia (Holguín, 1984, citado en Chona, 1998).

Por otra lado, la Biología participa también en la estrategia de higienizar a la población colombiana, pues desde 1886 la cuestión de la higiene era materia de administración pública. "La necesidad de mejorar las condiciones económicas del país estaban obviamente relacionadas con la higienización y las propuestas de emigración (ambas relacionadas con la degeneración de la raza). Hay que entender que la cuestión de la higiene fue sobre todo un imperativo educativo: educar es higienizar - No es la riqueza ni la prosperidad la garante social de la salud y del gobierno de la población sino más bien la inversa: un conjunto de prácticas que se encabalgan sobre el cuerpo sano asegurando su defensa contra la enfermedad, produciría los individuos que serían el fundamento de un orden social más sano y próspero" (Ospina, 1984).

Mientras en el Siglo XIX en el pensamiento occidental, la Biología define su objeto de conocimiento, rompiendo la continuidad existente entre el estudio de lo animado y lo inanimado, cuando las teorías sobre los seres vivos definen una metodología y unos principios, en la historia colombiana la existencia de la 
Bio-grafía Escritos sobre la Biología y su Enseñanza.

Edición Extra-Ordinaria.

Memorias del 1 Congreso Nacional de Investigación en Enseñanza de la Biología.

VI Encuentro Nacional de Investigación en Enseñanza de la Biología y la

Educación Ambiental. ISSN 2027 1034. P. p. 560-586.

Biología como discurso se da en las primeras décadas del siglo XX, pero ligada a las condiciones políticas y sociales, no como un discurso institucionalizado objeto de enseñanza. Se trata de una Biología muy ligada a la formación médica y a la acción política del estado.

Paralelamente se hacen publicaciones periódicas en el Instituto de Ciencias Naturales de la Universidad Nacional. La Biología se consolida poco a poco en las instituciones educativas con programas, metodologías y proyectos que superan levemente la inmediatez del control político y el desarrollo económico de la nación.

Una vez que el discurso biológico irrumpe en el ámbito universitario y es reconocido institucionalmente y con la influencia de misiones pedagógicas extranjeras, especialmente la proveniente de Alemania, se inicia la transformación de las escuelas normales en universidades formadoras de profesionales para la enseñanza, cumpliéndose así el proceso de institucionalización de la enseñanza de la biología en el sistema escolar colombiano

Hoy, a partir de la reorganización de las áreas obligatorias y fundamentales del conocimiento en la educación básica, se desdibuja y se descentra la atención a los procesos de comprensión e interpretación de lo vivo. El conocimiento biológico se refunde en un enfoque ambientalista, a pesar de que en el contexto universal ha tomado la forma de un poder que permite transformar la naturaleza y transformarse a sí mismo, suscitando discusiones por sus implicaciones éticas, políticas y hasta económicas.

Se resalta, que a pesar de la manifiesta institucionalización de la enseñanza de la Biología, aún hoy existen insuficiencias teóricas y falta una práctica pedagógica creada a partir de nuestros propios elementos culturales. Se supone que se han privilegiado los esquemas de racionalidad de sociedades, donde el saber biológico ha tenido una historia fecunda y la enseñanza ha sido objeto de reflexión permanente. Es urgente construir un espacio para pensar nuestra historia en relación con un sistema de enseñanza que debe crear nuevos modos de pensar la formación de sujetos en un contexto cultural propio.

Estos planteamientos pueden constituirse en base para renovar los procesos de formación desde una perspectiva epistemológica, e incluso darle una connotación distinta a la didáctica, al considerar no una historia sino las historias locales de las ciencias en relación con las didácticas con elementos como los siguientes:

- El conocimiento no surge de ideas abstractas, proposicionales, se construye en contextos socioculturales y tiene connotaciones ideológicas, en las que entra en juego el poder. 
Bio-grafía Escritos sobre la Biología y su Enseñanza.

Edición Extra-Ordinaria.

Memorias del 1 Congreso Nacional de Investigación en Enseñanza de la Biología.

VI Encuentro Nacional de Investigación en Enseñanza de la Biología y la

Educación Ambiental. ISSN 2027 1034. P. p. 560-586.

- Ubicar al sujeto humano como constructor de conocimiento, inserto en un contexto social que le genera situaciones que constituyen un sistema de significaciones y relacionar el desarrollo de sus procesos cognoscitivos con la epistemología de las ciencias, desde una historia propia, la nuestra.

- Darle sentido a la experiencia en la construcción de conocimientos, desde los mismos procesos cognoscitivos.

- La práctica vista como una serie de acciones que se constituyen en punto de partida para generar procesos de conocimiento.

- Concepciones de mundo que no se cuestionan y que condicionan la manera de comprender los fenómenos.

- El conocimiento no es universal, es diverso, cambia de acuerdo con el tipo de sociedad, con el tipo de cultura y con la época.

- Un conjunto de relaciones sociales, culturales y de poder que condicionan, los marcos de investigación y unas maneras diversas de pensar.

Es evidente que desde esta perspectiva incluso, se puede pensar en contribuir al reconocimiento de las culturas y de la diversidad en un país como Colombia que se considera biodiverso, pluriétnico y multicultural. Seguramente desde aquí puede surgir la interculturalidad como un elemento clave para la didáctica, como alternativa que reconoce otras formas de pensamiento, otras prácticas y otras formas de hacer.

\section{ENSEÑANZA DE LA BIOLOGÍA Y DIVERSIDAD.}

Una serie de investigaciones acerca del pensamiento infantil, realizadas en Colombia $^{3}$, hacen pensar que estamos ante retos muy significativos que demandan la capacidad de fijar nuestra atención en el otro; otro que tiene pensamiento propio y deseos propios e incluso en contravía de los nuestros y casi podría decirse opuestos a las concepciones culturales de las generaciones adultas dominantes. Se plantea que la escuela pierde significado para los niños si no logra una sólida vinculación con sus vidas, si no reconoce las permanentes transformaciones culturales en valores, estilos de relación, concepciones, sentidos y lenguajes que tienen lugar en su cotidianidad, más allá de los muros de la escuela (Castaño, 1998)

De la misma manera se considera que praxis como la formación de Licenciados en Biología que realiza la Universidad Pedagógica Nacional, en contextos

\footnotetext{
3 El pensamiento espontáneo de los niños acerca de lo vivo (Castaño, 2004); ¿Cómo piensan los niños lo vivo. Implicaciones para la enseñanza primaria (Castaño, NC., 1998); Ideas de los niños y niñas sobre la naturaleza. (Molina, A., 2005); Relaciones entre contexto cultural y explicaciones infantiles acerca de las adaptaciones vegetales (Molina, A. 2007); El niño indígena en su universo de ideas vivas. Pensamiento espontáneo de lo vivo de los niños (as) indígenas Piapoco en Básica primaria e implicaciones etno-didácticas. Peña, M.T. (2007).
} 
Bio-grafía Escritos sobre la Biología y su Enseñanza.

Edición Extra-Ordinaria.

Memorias del 1 Congreso Nacional de Investigación en Enseñanza de la Biología.

VI Encuentro Nacional de Investigación en Enseñanza de la Biología y la

Educación Ambiental. ISSN 2027 1034. P. p. 560-586.

campesinos mestizos e indígenas, que ponen la mirada en la concepción de un país multiétnico y pluricultural y en las condiciones de exclusión e inequidad de comunidades culturalmente diferenciadas, la violencia epistémica que se ejerce en las aulas y el antagonismo existente entre el conocimiento acerca de lo vivo, detentado por la Biología y los conocimientos ancestrales campesinos e indígenas, que también han demostrado ser valiosos y eficaces para la protección de la naturaleza, pueden constituirse en alternativas pertinentes para la enseñanza de la Biología (Castaño, 2009).

La educación principalmente, pero también los intereses políticos y económicos han impuesto el conocimiento biológico occidental como la única verdad. Como lo plantea Mato (2008, p. 105), de esta manera se contribuye a negar e invisibilizar nuestra condición pluricultural lo cual constituye un lastre histórico en términos de los desconocimientos que tenemos de nosotros mismos, lo cual también condiciona las posibilidades de construir desde la educación sociedades justas e incluyentes y el que cada una de estas sociedades pueda utilizar todos los saberes y talentos a su alcance para construir su presente y futuro "...este conflicto repercute también en la producción, circulación, apropiación y aplicación de conocimientos".

Afirma igualmente que tanto las propias preguntas de investigación, como los modos de producción de datos, dependen en última instancia de opciones epistemológicas, las cuales están asociadas a visiones del mundo y posiciones éticas y políticas que obedecen -entre otros factores- al tipo de relaciones que sostienen 0 aspiran sostener con actores sociales extraacadémicos. Las posiciones éticas y políticas son constitutivas del basamento epistemológico y de las perspectivas teóricas de las investigaciones; y así también de las preguntas y de los métodos.

Es importante para la enseñanza de la Biología involucrar un concepto semiótico de cultura (Geertz, 1996), pues la cultura es elemento constitutivo del pensamiento humano; los humanos construimos tramas de significaciones que constituyen acciones simbólicas necesariamente públicas. La intelección humana depende de la manipulación de recursos culturales de manera que se produzcan los estímulos mentales que se requieren para lograr un fin; es una búsqueda de información. La actividad mental determina principalmente la manera como una persona encara el mundo circundante. En este contexto, la acción del espíritu se desplaza desde la reunión de información sobre hechos del mundo exterior hacia la determinación significativa y el sentido emocional de esos hechos. Lo que interesa es no tanto resolver problemas sino clarificar sentimientos. Ello, aunque existan recursos culturales públicos es tan esencial en estos procedimientos, como lo es en el del razonamiento. No sólo las ideas sino también las emociones, dice, son artefactos 
Bio-grafía Escritos sobre la Biología y su Enseñanza.

Edición Extra-Ordinaria.

Memorias del 1 Congreso Nacional de Investigación en Enseñanza de la Biología.

VI Encuentro Nacional de Investigación en Enseñanza de la Biología y la

Educación Ambiental. ISSN 2027 1034. P. p. 560-586.

culturales en el ser humano; "un niño cuenta con sus dedos antes de contar "en el interior de su cabeza"; siente amor en su piel antes de sentirlo "en el corazón" .

La existencia de un sistema público de símbolos hace necesario cotejar estados y procesos; a este cotejamiento se le llama pensamiento reflexivo. La función del pensamiento reflexivo es "transformar una situación en la cual se experimenta oscuridad... de algún género, en una situación clara, coherente, ordenada, armoniosa" (Dewey. 1939. Citado en: Geertz).

En este contexto entonces, Geertz (1996; p. 88) afirma que la cultura "denota un esquema históricamente transmitido de significaciones representadas en símbolos, un sistema de concepciones heredadas y expresadas en formas simbólicas por medios con los cuales los hombres comunican, perpetúan y desarrollan su conocimiento y sus actitudes hacia la vida".

Al relacionar cultura, pensamiento y mente; el concepto mente no puede circunscribirse a conducta. En esta concepción mente designa una serie de disposiciones "una clase de habilidades, propensiones, facultades, tendencias, hábitos... la mente no es una acción ni una cosa es un sistema organizado de disposiciones que encuentra su manifestación en algunas acciones y en algunas cosas" (Geertz, 1996; p. 62-63). Lo cierto es que cuando atribuimos mente a una persona, no hablamos ni de sus acciones ni de sus productos en sí, sino que hablamos de su capacidad y su aptitud, de su disposición para realizar cierta clase de acciones y producir cierta clase de productos, capacidad y disposición que inferimos del hecho de que esa persona a veces cumple tales acciones y produce tales productos.

Desde esta perspectiva se considera que el símbolo designa "cualquier objeto, acto, hecho, cualidad o relación que sirva como vehículo de una concepción. Los símbolos son formulaciones tangibles de ideas, abstracciones de la experiencia fijadas en formas perceptibles, representaciones concretas de ideas, actitudes, juicios, anhelos o de creencias" (Geertz, 1996 p. 90). La concepción es el significado del símbolo y los actos culturales (aprehensión y utilización de las formas simbólicas) son hechos sociales públicos y observables.

El análisis de la cultura en consecuencia, debe ser interpretativo y en búsqueda de significaciones públicas, preguntando por su sentido y su valor. El propósito del enfoque semiótico de la cultura es "ayudarnos a lograr el acceso al mundo conceptual en el cual viven nuestros sujetos, de suerte que podamos, en el sentido amplio del término, conversar con ellos" (Geertz, 1996, p. 35)

La cultura pensada como conjunto de posibilidades; la educación como un hecho intencional, ubica al docente como un mediador cultural dada la realidad de una 
Bio-grafía Escritos sobre la Biología y su Enseñanza.

Edición Extra-Ordinaria.

Memorias del 1 Congreso Nacional de Investigación en Enseñanza de la Biología.

VI Encuentro Nacional de Investigación en Enseñanza de la Biología y la

Educación Ambiental. ISSN 2027 1034. P. p. 560-586.

confrontación tácita entre formas distintas y válidas de ver el mundo: la de los maestros y la de niños y adolescentes; la del pensamiento occidental y los conocimientos locales, constituyendo diferencias conceptuales complejas. La acción educativa es también una acción comunicativa, que confronta la vida cotidiana, que es dialógica, que es reconstructiva (Castaño, 1995).

Es necesario considerar el evento educativo en el contexto de la singularidad, del respeto por el otro, la responsabilidad con el otro. La educación debe construir un nosotros como sujeto y no un nosotros como objeto; como lo plantea Sartre (1993, pag 438) "el nosotros implica una pluralidad de subjetividades que se reconocen entre sí como tales... ser-para-el-otro (que) precede y funda al ser-con-el-otro".

Esto implica también que no hay un solo modo de conocer y en consecuencia no hay conocimientos más válidos que otros; solo hay conocimientos distintos. Algo es real desde el momento en que se encuentra en relación con nosotros. La historia de la Biología, como se verá enseguida, evidencia que lo que constituye la realidad es el sentido de nuestras experiencias, y no la estructura ontológica de los objetos; la realidad es del dominio de lo epistemológico, no de lo ontológico (Schutz, citado en: Mêlich, J.C. 1996).

\section{EN LA HISTORIA DE LA BIOLOGÍA, LO VIVO Y LA VIDA SE ENTRELAZAN ALGUNAS VECES.}

La historia de la Biología muestra que han existido distintas concepciones acerca de lo vivo: han estado entre el animismo, el antropocentrismo, el vitalismo y el materialismo. Para Smith (1975), se han dado en Occidente por lo menos cuatro biologías diferentes pero con sentido, la aristotélica, la cartesiana, la goethiana y la molecular. La biología aristotélica, afirma, es profundamente teleológica: la naturaleza no hace nada en vano, parece ser su fundamento. Para Descartes la imagen de lo vivo es un autómata; para Goethe lo vivo se constituye en una búsqueda de formas arquetípicas. Hoy lo vivo se concibe en términos moleculares y evolutivos.

El conocimiento cotidiano acerca de lo vivo también ha recorrido caminos distintos: una conciencia mágica que no marca límites entre lo subjetivo y lo objetivo, entre el yo y el no yo. El mundo es considerado en actividad dinámica y teleológica, lo mismo que un organismo vivo; además el ser humano se considera inmerso en la naturaleza, conectado a ella por hilos invisibles (Smith, 1975).

Tanto la historia de la Biología, como los saberes cotidianos acerca de lo vivo, muestran que las fuentes primarias del conocimiento biológico provienen de comunidades como los pescadores, agricultores, herbolarios, al tratar de resolver 
Bio-grafía Escritos sobre la Biología y su Enseñanza.

Edición Extra-Ordinaria.

Memorias del 1 Congreso Nacional de Investigación en Enseñanza de la Biología.

VI Encuentro Nacional de Investigación en Enseñanza de la Biología y la

Educación Ambiental. ISSN 2027 1034. P. p. 560-586.

las problemáticas de subsistencia (Jahn, 1990). De esta manera se piensa que el conocimiento acerca de lo vivo se origina también al tratar de desarrollar alternativas a problemas de subsistencia.

Las nociones y conocimientos acerca de las plantas, los animales y lo humano surgieron históricamente mucho antes de que existiese como tal cualquier tipo de ciencia y se encontraban articuladas al desarrollo de lo social, determinando quizás el desarrollo de la conciencia humana y seguramente impulsando no sólo el conocimiento racional acerca de lo que significa lo vivo, sino también la vivencia estética y emocional humana en lo que respecta a la naturaleza. Esa asignación de propiedades y correlaciones se entretejieron de forma compleja y muy arraigada con nociones de religión y fantasía (Jahn, 1990), lo que indica que las concepciones acerca de lo vivo han estado, a lo largo de la historia de la humanidad, entrelazadas a la vida misma.

La urgencia de sobrevivencia enseñó a los humanos, no solo a buscar afanosamente alternativas para solucionar problemas de la caza y la recolección, sino también a conocer la naturaleza, a diferenciar a los seres vivos de aquello que no lo es, a servirse de ellos o a evitarlos identificando sus propiedades. La adquisición de conocimientos sobre el entorno natural con su fauna y flora iba unida a la acción, estaba ligada a experiencias de las manifestaciones de vida propias del ser humano y llena de vivencias emocionales, afectivas y estéticas.

Las primeras nociones acerca del mundo circundante surgieron del arte y de este modo quizás el arte se convirtió en medio para la comprensión, a través de él se organizó el sistema de nociones acerca del mundo que nos rodea y que también empezó a regular y dirigir los procesos sociales y psíquicos, ordenando el caos, distribuyendo a los animales y plantas en clases de acuerdo con la urgencia de sobrevivir en medios agresivos y hostiles.

De la misma manera en la historia de las ciencias, como lo afirma Wuketits (1995), se muestra que tanto biólogos como filósofos de la biología defienden teorías particulares cuando definen el concepto vivo. Las teorías más difundidas se relacionan con el vitalismo y el mecanicismo. Los vitalistas sostienen que la vida y todas sus expresiones particulares, desarrollo, comportamiento, entre otras, dependen de agentes específicos que no pueden ser explicados en términos físicos y químicos. Estos agentes han tenido diferentes denominaciones a lo largo de la historia: entelequia, pneuma, vis plastica, principio vital. La teoría mecanicista, por su parte, se caracteriza por plantear que todo sistema viviente es un sistema mecánico, una clase de máquina basada en elementos químicos y que sigue las leyes universales de la materia. De ahí que no consideren diferencias notables entre los objetos vivos y no vivos puesto que los organismos son reducibles a compuestos moleculares. 
Bio-grafía Escritos sobre la Biología y su Enseñanza.

Edición Extra-Ordinaria.

Memorias del I Congreso Nacional de Investigación en Enseñanza de la Biología.

VI Encuentro Nacional de Investigación en Enseñanza de la Biología y la

Educación Ambiental. ISSN 2027 1034. P. p. 560-586.

En esta controversia subyace para Wuketits (1995), que respecto al pensamiento cognitivo hay dos tipos de biólogos. Aquellos que se inclinan al análisis y al reduccionismo y aquellos que por tener una intuición hacia la consideración del todo tienden a sintetizar más que analizar.

Pennock (2011) sostiene que la clasificación vida involucra elementos causales y pragmáticos. En lugar de buscar una definición única, fuerte, estas consideraciones sugieren que la vida es un concepto que agrupa (cluster), que tiene fronteras difusas y que hay múltiples formas legítimas de hacer la noción precisa para diferentes fines científicos. La definición de los conceptos científicos es un proceso de negociación que incluye tanto los intereses pragmáticos científicos como la estructura causal del mundo.

Tal vez esto significa que la vida es algo que reconocemos instintivamente, por eso no hay necesidad de palabras. Esto parece inverosímil, pero algunos biólogos han propuesto que la vida es así. Neil Campbell introduce el concepto de la siguiente

manera:

"La vida se resiste a una simple definición de una sola frase, ya que se asocia con numerosas propiedades emergentes. Sin embargo, casi cualquier niño percibe que un perro o un árbol está vivo y una roca no. Podemos reconocer la vida, sin definirla, y reconocemos la vida por lo que los seres vivos lo hacen" (Citado en Pennock, 2011, p.123)

Lovelock es más explícito, "Nuestro reconocimiento de los seres vivos es instantáneo y automático.. tenemos un programa altamente eficiente y rápido, para el reconocimiento de la vida en nuestro conjunto heredado de los instintos" (Citado en Pennock, 2011, p.123)

La forma en que juzgamos una distinción entre lo que es esencial y lo que no es esencial, será diferente cuando consideramos conceptos que son nociones culturales y conceptos como la vida que se refieren a características del mundo natural. De los conceptos científicos esperamos que haya una realidad que de forma a la gramática teórica. Sin embargo, que algo es un concepto científico o una clase natural no significa que no hay elementos sociales relevantes.

El análisis de las definiciones implícitas generales de vida se considera para Emmeche (1997), como base para la afirmación de que la biología como ciencia de los procesos de vida debe beneficiarse de la interdisciplinariedad y la búsqueda de principios universales de la organización.

Afirma que el origen del orden en el universo y el surgimiento de la organización se debe entender en un solo marco: causal, histórico y físico. La aparición de los 
Bio-grafía Escritos sobre la Biología y su Enseñanza.

Edición Extra-Ordinaria.

Memorias del 1 Congreso Nacional de Investigación en Enseñanza de la Biología.

VI Encuentro Nacional de Investigación en Enseñanza de la Biología y la

Educación Ambiental. ISSN 2027 1034. P. p. 560-586.

principios especiales de la organización biológica puede conceder autonomía conceptual a la biología, y podrá conceder al concepto de organismo, una ontología especial. Un concepto de vida en general es muy relevante en algunos contextos, por ejemplo, en el contexto de los discursos en general, la biología evolutiva, la protobiologia, la vida artificial, vida extraterrestre, filosofía de la biología y la bioética. Un concepto general de la vida biológica puede variar con los diferentes contextos; pero no hay que descartar, la generalidad.

El conocimiento de lo concreto debe implicar lo específico, así como también lo universal. Podríamos estar tentados a percibir 'el desorden de las cosas "(cf. Dupré, 1993) como un signo de imposibilidad del conocimiento general, pero no debemos resignarnos a aceptar el desorden del pensamiento; pues el desorden es inherente a la mente y la naturaleza. Esto no tiene por qué estar en conflicto con una crítica seria de cualquier forma de cientificismo, ya que la búsqueda de la universalidad de la ciencia no nos compromete con el universalismo en la política, la religión o la ética. Por otra parte, la universalidad de la ciencia (como un valor, o como un signo de la modernidad) no es lo mismo que la universalidad en la política o en la cultura, plantea Emmeche (1997).

\section{LA ENSEÑANZA DE CONCEPTOS BIOLÓGICOS Y SU RELACIÓN CON LA VIDA.}

En Brasil (Correa, sf.) plantea que aunque la enseñanza de las Ciencias Naturales está organizada en gran parte alrededor de conceptos biológicos, hay dificultades en la conceptualización de la vida; también la encuentran en los medios científicos en donde consideran que no hay necesidad de tal conceptualización o que es muy difícil capturar en un esquema conceptual la diversidad de fenómenos de vida pues tienen varias facetas y distintos niveles de organización.

Las definiciones de vida raramente se discuten en profundidad, ni siquiera se mencionan en los libros de texto, afirma Emmeche (1997), aludiendo que esto es reflejo de una biología empirista tradicional, que solo toma en cuenta los hechos y desde la que se argumenta que no hay una definición clara de la vida, el concepto no se puede definir; la definición del concepto no es importante para la Biología.

En una investigación acerca de la percepción de estudiantes en Turquía, acerca de la biodiversidad (Nurettin, 2008), los resultados muestran que aunque las opiniones de los alumnos hacia los seres vivos y la naturaleza eran similares a una comprensión holística, sus construcciones acerca de temas como la relación entre la nutrición y el flujo de energía eran débiles, y la idea de la visión antropocéntrica en la que los seres humanos están en el centro de todos los seres vivos está muy extendida entre ellos. Se evidenció también que los estudiantes, tenían "ceguera" a las plantas, no las reconocían como seres vivos. Lindeman- 
Bio-grafía Escritos sobre la Biología y su Enseñanza.

Edición Extra-Ordinaria.

Memorias del 1 Congreso Nacional de Investigación en Enseñanza de la Biología.

VI Encuentro Nacional de Investigación en Enseñanza de la Biología y la

Educación Ambiental. ISSN 2027 1034. P. p. 560-586.

Matthies (2002, citado en Nurettin, 2008) encuentran igual que Nurettin (2008) que los seres vivos más apreciados por los estudiantes eran las mascotas (gatos, perros y caballos) y las especies exóticas, como delfines, tigres y leones.

Nurettin (2008) concluye que la construcción cognitiva del concepto vida ocurre asociándolo con los animales. Además de acuerdo con los resultados, el primer ser viviente al que se asocia el concepto vida es el humano.

Bardel (1997, citado en Nurettin, 2008) sugiere que los estudiantes construyen el concepto de vida asociándolo al concepto de movimiento y argumenta que esto es de carácter animístico, considerándolo como un error conceptual. Afirma que el concepto de movimiento está entre las razones más importantes por las cuales la gente muestra más interés en los animales que en las plantas (Wandersee, 1986; Kinchin, 2000. Citados en Nurettin, 2008).

\section{LOS RETOS}

De acuerdo a lo planteado los retos de la educación en biología, están en establecer relaciones entre las formas políticas contemporáneas, la educación y la búsqueda de alternativas posibles en contexto. de lo que somos - nosotros y los otros. Gestar propuestas alternativas que involucren a todos los sectores de la sociedad, como un ejercicio de libertad y solidaridad humana. Abordar la globalización, el posdesarrollo y la sustentabilidad ecológica, en formas social y políticamente efectivas.

Para lograrlo es fundamental auto reconocernos como sujetos de poderes: reconocernos en y desde la diferencia evita anular la mirada del otro y también rompe la inercia de colonizar epistemológicamente a las culturas y grupos humanos definidos en la alteridad; perder las certezas y ganar libertades, no exponernos y exponer a los distintos tipos de violencias que genera la reproducción sin sentido y la homogenización de una sola forma de concebir el mundo.

Preguntarnos si la lógica formal que sustenta el pensamiento científico es la única forma de entender la idea de verdad. Compartir con otros modos de saber y construir puentes epistémicos entre diferentes rutas para conferir sentido a la experiencia en la medida en que lo global y lo local se articulan en la forma que el sujeto significa su experiencia y el contexto.

"Tenemos que recurrir a una forma de conocimiento que no reduzca la realidad a aquello que existe...una forma de conocimiento que aspire a una concepción expandida de realismo, que incluya realidades suprimidas, silenciadas 0 marginadas, tanto como realidades emergentes o imaginadas" (Santos, 2009). 
Bio-grafía Escritos sobre la Biología y su Enseñanza.

Edición Extra-Ordinaria.

Memorias del I Congreso Nacional de Investigación en Enseñanza de la Biología.

VI Encuentro Nacional de Investigación en Enseñanza de la Biología y la

Educación Ambiental. ISSN 2027 1034. P. P. 560-586.

Apostar por un pluriverso donde todo particularismo tenga cabida para mejorar las condiciones de vida de las comunidades, democratizar el conocimiento, reconocer los saberes locales, otras formas de percepción y producción de conocimiento, otras epistemologías y cosmogonías, diversas lógicas y diversas estéticas (Castaño, 2009).

En este cuestionamiento inciden aspectos como:

- El antagonismo existente entre el conocimiento acerca de lo vivo, detentado por la Biología y los conocimientos ancestrales indígenas, que también han demostrado ser valiosos y eficaces.

- La acción pedagógica demanda actitudes en el docente para convertirse en orientador y dinamizador de procesos de aprendizaje, que atiendan las distintas cosmovisiones, lo cual exigiría que el aula pase de ser espacio de discriminación y de sanción ${ }^{4}$, de transmisión y repetición, a constituirse en lugar de encuentro y de construcción intersubjetiva y en consecuencia intercultural.

- La necesidad de transformar las preguntas que invariablemente guían la educación y reconocer diversas fuentes de conocimiento.

- Considerar que la razón está influenciada por la cultura; hay tantas verdades como culturas, clases sociales, intereses y creencias existan.

- La contribución que se puede hacer a la formación de docentes en Biología a la construcción de un país diverso biológicamente y culturalmente.

- La educación principalmente, pero también los intereses políticos y económicos han impuesto el conocimiento biológico occidental como la única verdad.

La formación docente en los países latinoamericanos, plantea por su parte Nuñez (2004) "se encuentra inmersa en una serie de complejidades y contrariedades. Los educadores, como formadores de una sociedad, reproducen sin pausa e inconscientemente, en su mayoría, los saberes modernos deudores de la cultura occidental. Sin embargo, los contextos de la acción docente exhiben una gran riqueza acumuladas en las sabidurías de los pueblos que son ignoradas en los programas escolares formales. Se propone la necesidad urgente de otorgar mayor pertinencia cultural y social a los saberes que se enseñan desde la escuela.

\footnotetext{
${ }^{4}$ Se habla de discriminación y de sanción porque en el aula hoy se trata esencialmente de determinar quiénes son capaces y quienes no, para asignarles premios o castigos en forma de logros positivos o negativos, respectivamente.
} 
Bio-grafía Escritos sobre la Biología y su Enseñanza.

Edición Extra-Ordinaria.

Memorias del 1 Congreso Nacional de Investigación en Enseñanza de la Biología.

VI Encuentro Nacional de Investigación en Enseñanza de la Biología y la

Educación Ambiental. ISSN 2027 1034. P. p. 560-586.

Para ello dice, son claves dos aspectos: otorgar un estatus académico a los saberes locales, potenciados por las epistemes emergentes ante la crisis de la modernidad y establecer un diálogo intercultural entre el saber local y el universal para aprovechar sus fortalezas asumiendo una postura de sinergismo de los saberes humanos.

Es evidente que desde esta perspectiva incluso, se puede pensar en contribuir al reconocimiento de las culturas y de la diversidad en un país como Colombia que se considera biodiverso, pluriétnico y multicultural. Seguramente desde aquí puede surgir la interculturalidad como un elemento clave para la didáctica, como alternativa que reconoce otras formas de pensamiento, otras prácticas y otras formas de hacer.

Es así como Santos (2009, pág. 50) afirma que el paradigma dominante es totalitario pues es un modelo global de racionalidad científica que admite variedad interna, pero pone fronteras al sentido común y a las humanidades. "Consagra al hombre como sujeto epistémico, pero lo expulsa en cuanto sujeto empírico".

Constituye un modelo totalitario que niega el carácter racional a todas las formas de conocimiento que no se pautan por sus principios epistemológicos y reglas metodológicas. Desconfía sistemáticamente de las evidencias de nuestra experiencia inmediata: privilegia el cómo funciona de las cosas en detrimento de cuál es el agente o cuál es el fin de las cosas. "Mientras en el sentido común y por lo tanto en el conocimiento práctico en que él se traduce, la causa y la intención conviven sin problemas, en la ciencia la determinación de la causa formal se obtiene expulsando la intención" (Santos, 2009, p.21).

Propone como alternativa epistemológica, un conocimiento intersubjetivo, descriptivo y comprehensivo: "En vez de la eternidad, la historia; en vez del determinismo, la imprevisibilidad; en vez del mecanicismo, la interpenetración, la espontaneidad y la autoorganización; en vez de la reversibilidad, irreversibilidad y la evolución; en vez del orden, el desorden; en vez de la necesidad, la creatividad y el accidente. El paradigma emergente "Un conocimiento prudente para una vida decente" que tiene como elemento principal que todo el conocimiento científico natural es científico social: vocación holística que interrelaciona los conceptos de ser humano, cultura y sociedad. Los fenómenos naturales se estudian como si fueran fenómenos sociales. Además:

- Todo el conocimiento es local y total: tiene como horizonte la totalidad universal o la totalidad indivisa; se constituye alrededor de temas que son adoptados por grupos sociales con proyectos de vida locales. La fragmentación no es disciplinar, es temática. La ciencia se acepta como 
Bio-grafía Escritos sobre la Biología y su Enseñanza.

Edición Extra-Ordinaria.

Memorias del 1 Congreso Nacional de Investigación en Enseñanza de la Biología.

VI Encuentro Nacional de Investigación en Enseñanza de la Biología y la

Educación Ambiental. ISSN 2027 1034. P. p. 560-586.

analógica y se asume como traductora: generaliza a través de la cualidad y la ejemplificación.

- Todo conocimiento es autoconocimiento: "El acto de conocimiento y el producto de conocimiento son inseparables...el objeto es la continuación del sujeto por otros medios. Los presupuestos metafísicos, los sistemas de creencias, los juicios de valor no están ni antes ni después de la explicación científica de la naturaleza o de la sociedad. Son parte integrante de esa misma explicación. La ciencia moderna es un juicio de valor, es autobiográfica".

- Todo el conocimiento científico busca constituirse en sentido común: La ciencia moderna produce conocimientos y desconocimientos. Ninguna forma de conocimiento es en sí misma racional; sólo la configuración de todas ellas es racional... el sentido común enriquece nuestras relaciones con el mundo: hace coincidir causa e intención, le subyace una visión de mundo basada en la acción y en el principio de la creatividad y de la responsabilidad individuales. Es práctico y pragmático; es transparente y evidente, es retórico y metafórico; no enseña, persuade...interpenetrado por el conocimiento científico puede estar el origen de una nueva racionalidad. Una racionalidad hecha de racionalidades".

Afirma la dualidad entre dos formas de conocimiento:

- El conocimiento - emancipación: el momento del saber es la solidaridad, el reconocimiento del otro como igual e igualmente productor de conocimiento. La solidaridad no tiene lugar en el conocimiento científico.

- El conocimiento - regulación. Constituye una forma hegemónica, conocemos creando orden; la regulación social

Es necesario optar desde la educación en uno u otro sentido. Ello implica de una u otra manera pasar del pensamiento único a la participación activa: gestar propuestas alternativas que involucren a todos los sectores de la sociedad, como un ejercicio de libertad y solidaridad humana y abordar la globalización, el posdesarrollo y la sustentabilidad ecológica, en formas social y políticamente efectivas.

Para lograrlo es fundamental auto reconocernos como sujetos de poderes. Reconocernos en y desde la diferencia evita anular la mirada del otro y también rompe la inercia de colonizar epistemológicamente a las culturas y grupos humanos definidos en la alteridad; perder las certezas y ganar libertades, no exponernos y exponerlos a los distintos tipos de violencias que genera la reproducción sin sentido y la homogenización de una sola forma de concebir el mundo. 
Bio-grafía Escritos sobre la Biología y su Enseñanza.

Edición Extra-Ordinaria.

Memorias del 1 Congreso Nacional de Investigación en Enseñanza de la Biología.

VI Encuentro Nacional de Investigación en Enseñanza de la Biología y la

Educación Ambiental. ISSN 2027 1034. P. p. 560-586.

Compartir con otros modos de saber y construir puentes epistémicos entre diferentes rutas para conferir sentido a la experiencia en la medida en que lo global y lo local se integran en la forma que el sujeto significa su experiencia y el contexto.

Apostar por un pluriverso donde todo particularismo tenga cabida para mejorar las condiciones de vida de las comunidades, democratizar el conocimiento, reconocer los saberes locales, otras formas de percepción y producción de conocimiento, otras epistemologías y cosmogonías, diversas lógicas y diversas estéticas (Castaño, 2009)

El debate por el reconocimiento de otros conocimientos para el sistema educativo parte por desmontar la violencia epistémica en la medida que esta opera como un dispositivo de poder, de regulación y clasificación de lo que se debe enseñar. Esto nos puede dar como resultado la construcción de un habitus que se revele contra la imposición del conocimiento y la asimilación de esta imposición, es decir un habitus no construido para la obediencia sino, para la liberación, en este caso del sistema educativo que legitima un tipo especifico de conocimiento sobre la base de la negación de otros conocimientos (Albán, 2010).

Igualmente plantea Santos (2009) que nuestra situación es algo compleja: podemos afirmar que tenemos problemas modernos para los cuales no tenemos soluciones modernas. $Y$ esto le da a nuestro tiempo el carácter de transición: tenemos que hacer un esfuerzo muy exigente por reinventar la emancipación social. Nuestro primer problema para la gente que vive en el Sur es que las teorías están fuera de lugar, no se adecuan realmente a nuestras realidades sociales. Vivimos un problema complicado, una discrepancia entre teoría y práctica social que es dañina para la teoría y también para la práctica. Para una teoría ciega, la práctica social es invisible; para una práctica ciega, la teoría social es irrelevante. No es simplemente un conocimiento nuevo lo que necesitamos; necesitamos un nuevo modo de producción de conocimiento. No necesitamos alternativas, necesitamos un pensamiento alternativo de las alternativas.

García Canclini (2004, p. 53), llama la atención en que es necesario articular tres procesos: las diferencias, las desigualdades y la desconexión. "Hay aspectos que en la conformación de las comunidades son innegociables e inasimilables. Para millones, el problema no es mantenerse separados sino ser incluidos, llegar a conectarse sin que se atropelle su diferencia, ni se los condene a la desigualdad. Podemos conectarnos con los otros, únicamente para obtener información, como lo haríamos con una máquina proveedora de datos. Conocer al otro, en cambio, es tratar con su diferencia". 
Bio-grafía Escritos sobre la Biología y su Enseñanza.

Edición Extra-Ordinaria.

Memorias del 1 Congreso Nacional de Investigación en Enseñanza de la Biología.

VI Encuentro Nacional de Investigación en Enseñanza de la Biología y la

Educación Ambiental. ISSN 2027 1034. P. p. 560-586.

Bourdieu (sf) también se refiere al hecho que las formas de exclusión de los otros, evidenciadas en el racismo son probablemente las más sutiles, las más difíciles de reconocer, y las que menos se denuncian. A esto lo denomina racismo de la inteligencia .

Alude que el racismo es propio de una clase dominante cuya reproducción depende, en parte, de la transmisión del capital cultural, un capital heredado cuya propiedad es la de ser un capital incorporado, pero aparentemente natural, genético. El racismo de la inteligencia es aquello por lo cual los dominantes tratan de producir una "teodicea de su propio privilegio ${ }^{5 "}$ como dice Weber (citado en Bourdieu, sf), esto es, una justificación del orden social que ellos dominan. Es lo que hace que los dominantes se sientan justificados de existir como dominantes, que sientan que son de una esencia superior. Todo racismo es un esencialismo y el racismo de la inteligencia es la forma de sociodicea característica de una clase dominante cuyo poder se ubica en la posesión de títulos que, como los académicos, son supuestas garantías de inteligencia con los que se accede hoy a posiciones de poder social y económico autojustificatorias de privilegios que se traducen en construcciones simbólicas de superioridad.

Algunas de las propiedades de este racismo se deben también a que las censuras en relación con las formas de su expresión se han reforzado, por lo cual sólo puede expresarse en formas muy eufemizadas, la más común, es el aparente carácter científico del discurso. Si se invoca el discurso científico para justificar el racismo de la inteligencia, esto no se debe sólo a que la ciencia representa la forma dominante del discurso legítimo, también a que es un poder que cree estar fundado en la ciencia, como lo es el tecnocrático, recurre naturalmente a ella para fundar su poder. Se debe a que la inteligencia es la que legitima para gobernar cuando el gobierno se dice fundado en la ciencia y en la competencia "científica" de los gobernantes. La ciencia es cómplice de todo lo que le piden que justifique, como forma dominante de representación del mundo, con su discursividad y sus regímenes de poder.

Para Bourdieu (1999, p.75-76) "la verdad científica reside en una especie particular de condiciones de producción; más precisamente, en un estado determinado de la estructura y del funcionamiento del campo científico. El universo 'puro' de la ciencia más 'pura' es un campo social como cualquier otro, con sus relaciones de fuerza y sus monopolios, sus luchas y sus estrategias, sus intereses y sus beneficios, pero donde todos estos invariantes revisten formas específicas."

Es así como se plantean unas condiciones para la enseñanza de la Biología, especialmente cuando se piensa en un país biodiverso, pluriétnico y multicultural, que implican no sólo conocimiento sino también reflexión y comprensión y, sobre

${ }^{5}$ Seguramente a manera de justificación de si, como si fuera natural. 
Bio-grafía Escritos sobre la Biología y su Enseñanza.

Edición Extra-Ordinaria.

Memorias del I Congreso Nacional de Investigación en Enseñanza de la Biología.

VI Encuentro Nacional de Investigación en Enseñanza de la Biología y la

Educación Ambiental. ISSN 2027 1034. P. p. 560-586.

todo, visión de mundo presta a modificarse y a transformarse a partir de estas relaciones:

a. Un proceso de construcción colectiva de conocimientos y saberes en torno a problemáticas en contextos diversos, proponiendo alternativas de pertenencia territorial.

b. Comprensión de la compleja manifestación de la vida y la reconsideración de las relaciones ecosistema-cultura - biopolítica, que requieren de la reconfiguración de las concepciones acerca del conocimiento científico y biológico en particular, al igual que de su enseñanza, partiendo del presupuesto que lo vivo y la vida no son campos separados. De tal manera que de lo vivo sea una preocupación de la biología y la vida de otras disciplinas. Se trata de desarrollar un enfoque sistémico y complejo, de construir puentes entre las disciplinas e incluso ir más allá de estas, involucrando por ejemplo, los conocimientos ancestrales de pueblos que han sabido conservar la naturaleza durante siglos y construir un diálogo intercultural.

c. Otras epistemologías acerca del conocimiento biológico buscan articular el estudio de lo vivo con aspectos sociales y culturales, e incluso con conceptos de desarrollo económico. En esa medida no es posible pensar la enseñanza de la biología sólo desde la óptica del pensamiento analítico y de la educación formal escolar.

d. Una ruptura de la relación saber/poder, a partir de la transformación de las representaciones academicistas, democratizando el conocimiento biológico y poniéndolo a la disposición de las comunidades, independientemente de sus niveles educativos, por la trascendencia que implica este conocimiento, en la vida de las personas, de las sociedades y de la construcción de un país como el nuestro. Desplazando los paradigmas de control, de dominación y homogenización que no constituyen alternativas a los procesos de globalización, sino que por el contrario, contribuyen a la invisibilización y a la subordinación de los sujetos, llevando a una violencia de las mentalidades.

Se considera así que es vital comprometerse como academia con realidades concretas, a partir de la comprensión de las particularidades del contexto y de los vínculos con las poblaciones, en la perspectiva de trascender con sentido las posibilidades de vida de las personas y de las comunidades, no desde la lógica del desarrollo, el crecimiento económico y el consumismo, sino desde paradigmas alternativos que constituyen otras mentalidades, otras visiones de mundo y otras formas de vida (Castaño, 2010).

Lo anterior implica el reconocimiento de diversas epistemologías, cosmovisiones, 
Edición Extra-Ordinaria.

Memorias del I Congreso Nacional de Investigación en Enseñanza de la Biología.

VI Encuentro Nacional de Investigación en Enseñanza de la Biología y la

Educación Ambiental. ISSN 2027 1034. P. p. 560-586.

éticas y estéticas, e igualmente propiciar la autonomía de los saberes locales en la construcción y producción de conocimiento mediante la comprensión de las distintas manifestaciones culturales, formas de conservación de la naturaleza, dinámicas sociales y procesos económicos y políticos.

Además supone establecer consensos y fortalecer vínculos con las instituciones, grupos sociales y personas con quienes se construyen los proyectos educativos; de manera que se proyecten hacia su reconocimiento y se contribuya a generar procesos ecológica y socialmente sustentables. Seguramente así, desde la enseñanza de la Biología se podrá a contribuir a la construcción de un país más justo e incluyente.

\section{BIBLIOGRAFÍA}

- ABELL, S. y LEDERMAN, N. 2007. Handbook of research on science education. Tomo 1. Routledge: New York.

- ALBÁN, A. 2010. Seminario Educación, Diversidad e Integración. Doctorado interinstitucional en Educación. Universidad Pedagógica Nacional.

- BEILLEROT, J. y otros. 1998. Saber y relación con el saber. Paidós: Buenos Aires.

- BERSTEIN, B. 1990. Clases, códigos y control: la estructura del discurso pedagógico. Madrid: Morata.

- BODNAR, Y. 2005. Apuntes sobre la diversidad cultural y la información sociodemográfica disponible sobre los pueblos indígenas de Colombia. En: Notas de Población, Año XXXI, núm. 79, Naciones Unidas, CEPAL, Santiago de Chile.

- BOURDIEU, P. (SF). El racismo de la inteligencia. http://www.scribd.com/doc/7249571/Bourdieu-Pierre-El-Racismo-de-LaInteligencia

- BOURDIEU, P.; PASSERON, J. C. 2005. La reproducción. Elementos para una teoría del sistema de enseñanza. Fontamara:Mexico.

- BOURDIEU, Pierre. 1997. Razones prácticas. Anagrama: Barcelona,

- BOURDIEU, Pierre. 1999. "El campo científico", en Intelectuales, política y poder. Eudeba, Buenos Aires.

- CAMILLONI, A.; BASABE, L.,FEENEY, S. 2007. El saber didáctico. Buenos Aires: Paidós

- CASTAÑO CUÉLLAR, N.C. 1995. La educación en el contexto social, cultural y político contemporáneo. Ensayo no publicado.

- CASTAÑ̃o, N. C. (2009). Construcción social de universidad para la inclusión: la formación de maestros con pertinencia y en contexto, desde una perspectiva intercultural [Colombia] pp. 183 - 206. En: Mato, Daniel. 
Bio-grafía Escritos sobre la Biología y su Enseñanza.

Edición Extra-Ordinaria.

Memorias del 1 Congreso Nacional de Investigación en Enseñanza de la Biología.

VI Encuentro Nacional de Investigación en Enseñanza de la Biología y la

Educación Ambiental. ISSN 2027 1034. P. p. 560-586.

Educación Superior, Colaboración Intercultural y Desarrollo Sostenible/Buen Vivir. UNESCO - IESALC.

- CASTAÑO, N.C.; LEUDO, M. Las nociones de los niños acerca de lo vivo. Implicaciones didácticas. En: Revista TED. Universidad Pedagógica Nacional. 1998, No. 4. Pp 42-49

- CASTAÑO, Norma Constanza. 2009. Los sujetos y el saber. La formación de sujetos de saber en escenarios culturales diversos. Documento sin publicar.

- CASTAÑO, NORMA CONSTANZA; PAOLA GAMBOA; DIANA PACHECO y otros. (2007) Educación Rural y Diversidad. En: Reflexiones acerca de la Investigación en Educación y Pedagogía. Bogotá: Universidad Pedagógica Nacional, págs. 195-218

- CHONA, G.; CASTAÑO, N.C.; CABRERA, F.; y otros. "Lo que nos dice la historia de las enseñanza de la biología en Colombia- Una aproximación". En: Revista Tecne, Episteme y Didaxis. 1998, No 4 pp 5-10

- CORREA, A.; MEGLHORATTI, A.; DE ANDRADE, A. SF. Conceito de vida: uma proposta para o ensino de ciencia na educacao fundamental. En: http://www.fae.ufmg.br/abrapec/viempec/7enpec/pdfs/610.pdf. Revisado 24/08/2011

- DUPRÉ, J., 1993: The Disorder of Things. Metaphysical Foundations of the Disunity of Science. Cambridge, Massachusetts: Harvard University Press.

- EMMECHE, Claus, Defining Life, Explaining Emergence. On-line paper, 1997. <http://www.nbi.dk/ emmeche/cePubl/97e.defLife.v3f.html>.Revisado Octubre de 2010.

- GARCíA CANCLINI, H. 2004. Diferentes, desiguales y desconectados. Mapas de la interculturalidad. Gedisa Ed. España

- GARCÍA MARÍN, JORGE. Educación y reproducción cultural: el legado de Bourdieu. En: Práxis Educacional Vitória da Conquista n. 2 p. 71-77 2006

- GEERTZ, Cliford. 1996. La interpretación de las culturas. Barcelona: Gedisa

- GUTIERREZ, ALICIA. Poder, hábitus y representaciones: recorrido por el concepto de violencia simbólica en Pierre Bourdieu. En: Revista Complutense de Educación Vol. 15 Núm. 1 (2004) 289-300

- HERNÁNDEZ, ASTRID, PINILLA DAVID Visibilización de la población étnica en el Censo general de 2005: análisis comparativo de los principales indicadores demográficos.

http://www.dane.gov.co/revista ib/html r8/articul05.html Consultado en Febrero de 2011.

- JAHN, I. LÖTHER, R., SENGLAUB, K. 1990. Historia de la Biología : teorías, métodos, instituciones y biografías breves. Ed. Labor: Madrid

- LEFF, ENRIQUE,. 1986. Los problemas del conocimiento y la perspectiva ambiental del desarrollo, Ed. Siglo XXI, México, p. 36

- McNEIL, L.M. (2000). Creating new inequalities: contradictions of reform. Phi Delta Kappan,81, 729-734. 
Edición Extra-Ordinaria.

Memorias del I Congreso Nacional de Investigación en Enseñanza de la Biología.

VI Encuentro Nacional de Investigación en Enseñanza de la Biología y la

Educación Ambiental. ISSN 2027 1034. P. p. 560-586.

- MATO, DANIEL. No hay saber "universal", la colaboración intercultural es imprescindible. En: Alteridades, 2008. 18 (35): Págs. 101-116.

- MÊLICH, J.C 1996. Del extraño al cómplice. La educación en la vida cotidiana. Barcelona:Anthropos.

- MOLINA, A. 2010. Consideraciones sobre la enseñanza de las ciencias y el contexto cultural. Documento sin publicar.

- NÚÑEZ, J. formación docente y saberes locales: una mirada desde la ruralidad. Revista Digital eRural, Educación, cultura y desarrollo rural. Año 2 $\mathrm{N}^{\circ} \quad 3 \quad$ Junio $2004, \quad$ ISSN $0717-9898$. http://educacion.upla.cl/revistaerural/JNR2.htm

- NURETTIN Y., HALIL A.; UGULU, I, DOGAN, Y. An investigation on students' perceptions of biodiversity. En: Natura montenegrina, podgorica, 7(3), 2008; pp 176-184

- OSPINA, A La higiene y la escuela. Principio de siglo XX en Colombia Tesis de grado Universidad del Valle. -Facultad de Filosofía Cali. 1984

- PENNOCK, Robert. Negotiating boundaries in the definition of life: Wittgensteinian and Darwinian insights on resolving conceptual border conflicts. En: Synthese. Publicado online 15 febrero de 2011 por Springer Science+Business Media B.V. 2011. Consultado en marzo 2011.

- PEÑA, Marco Tulio. 2007. El niño indígena en su universo de ideas vivas. Pensamiento espontáneo de lo vivo de los niños (as) indígenas Piapoco en Básica primaria e implicaciones etno-didácticas. Trabajo de grado Licenciatura en Biología. Universidad Pedagógica Nacional.

- RESTREPO, Olga. 1984. Apuntes para una historia social de la Biología en Colombia. Colciencias. Bogotá.

- SANTOS, BOAVENTURA DE SOUSA. 2009. Una epistemología del Sur: la reinvención del conocimiento. Siglo XXI-. Clacso coediciones: Mexico

- SARTRE, Jean Paul. 1993. El ser y la nada: Ensayo de ontología fenomenológica. Altaya :Madrid. p. 438

- SMITH, C.U.M. 1975. El problema de la vida. Ensayo sobre los orígenes del conocimiento biológico. Alianza Universidad: Madrid

- WUKETITS. F.M. The history of the concept of life: a game of mind. En: Revista Ludus vitalis. Vol III. No 4, 1995. Pp 39-49

- ZAMBRANO, ARMANDO. 2010. Seminario de Pedagogía y Didáctica. Doctorado Interinstitucional en Educación. 